\title{
The magnetically collimated jet of the evolved star W43A
}

\section{W. H. T. Vlemmings *† $^{* \dagger}$}

Jodrell Bank Observatory, Univ. of Manchester, Macclesfield, Cheshire SK11 9DL, U.K.

E-mail: wouter@jb.man.ac.uk

\section{P. J. Diamond}

Jodrell Bank Observatory, Univ. of Manchester, Macclesfield, Cheshire SK11 9DL, U.K.

\section{H. Imai}

Department of Physics, Faculty of Science, Kagoshima University, Kagoshima 890-0065, Japan

\begin{abstract}
We present Very Long Baseline Array (VLBA) observations of the linear and circular polarization of the $\mathrm{H}_{2} \mathrm{O}$ masers in the collimated jet of W43A. The observations reveal a strong toroidal magnetic field, indicating that the jet is magnetically collimated. These are the first direct measurements of the magnetic field strength and direction in a collimated jet from an evolved star on its way to become a planetary nebula. The magnetic field strength in the jet extrapolated back to the stellar surface is fully consistent with the magnetic fields determined from $\mathrm{SiO}, \mathrm{H}_{2} \mathrm{O}$ and $\mathrm{OH}$ maser polarization in a large sample of evolved stars. The origin of the magnetic field is yet unknown, although the jet precession might point to the existence of a heavy planet or stellar companion. Our observations thus provide strong evidence for magnetic collimation in the jets, that likely play an important role in shaping planetary nebulae.
\end{abstract}

8th European VLBI Network Symposium

September 26-29, 2006

Toruń, Poland

\footnotetext{
* Speaker.

${ }^{\dagger}$ WV was supported by a Marie Curie Intra-European fellowship within the 6th European Community Framework Program under contract number MEIF-CT-2005-010393.
} 


\section{Introduction}

W43A is an evolved star at a distance of $2.6 \mathrm{kpc}$ [1]]. It is surrounded by a thick circumstellar envelope (CSE) that exhibits $\mathrm{OH}, \mathrm{H}_{2} \mathrm{O}$ and $\mathrm{SiO}$ masers ([2] and references therein). The $\mathrm{H}_{2} \mathrm{O}$ masers of W43A occur in two clusters at $\sim 1000$ AU from the star near the opposing tips of a collimated jet. The jet, with a velocity of $145 \mathrm{~km} \mathrm{~s}^{-1}$, has an inclination of $39^{\circ}$ with respect to the sky plane, and a position angle of $65^{\circ}$. It precesses by $5^{\circ}$ with a period of 55 years. The dynamical age of the jet is inferred to be only approximately 50 years [3]. W43A is interpreted as belonging to a class of objects undergoing a rapid transition from an evolved star into a planetary nebula ( $\mathrm{PNe}$ ). Currently, owing to their short expected lifetime of less than 1000 years, only 5 sources of this class have been identified [3, 4, 5, 6].

Maser polarization observations have revealed the magnetic field throughout the CSEs of a large number of evolved stars. Close to the central star, at typically $\sim 2$ stellar radii, SiO masers indicate ordered fields of the order of several Gauss (e.g. [7. 8]). At the outer edge of the CSE, the polarization measurements of $\mathrm{OH}$ masers reveal milliGauss magnetic fields and indicate weak alignment with CSE structure [9]. Recently, Zeeman splitting measurements of $\mathrm{H}_{2} \mathrm{O}$ masers in the CSEs of a sample of evolved stars revealed large scale magnetic fields with field strengths between a hundred milliGauss up to a few Gauss [10, 11]. While the origin of the magnetic field is still unclear, theoretical models have shown that a dynamo between the slowly rotating stellar outer layers and the faster rotating core can produce the observed magnetic fields [12]. However, the required additional source of angular momentum to maintain magnetic field likely requires the presence of a binary companion or heavy planet [13, 14, 15].

Magnetic fields around evolved stars are thought to be one of the main factors in shaping the CSEs and producing the asymmetries during the evolution of a spherically symmetric star into the often asymmetric PNe. Similar to well-established theories of collimated outflows in young stellar objects, theoretical models show that magnetic fields could be the collimating agents of the bi-polar jets in young proto-planetary nebulae such as W43A (e.g. [16]). Here we describe the first direct detection of a magnetically collimated jet from the evolved star W43A, recently published in [17] (hereafter V06).

\section{Observations}

The observations of W43A were done with the NRAO ${ }^{1}$ VLBA on 8 December 2004 at the frequency of the $6_{16}-5_{23}$ rotational transition of $\mathrm{H}_{2} \mathrm{O}, 22.235080 \mathrm{GHz}$. The correlation and data reduction was carried out as described in [18], where similar methods were used to study $\mathrm{H}_{2} \mathrm{O}$ maser magnetic fields in a high-mass star-forming region. Polarization calibration was done with respect to the calibrator $\mathrm{J} 1743-0350$ and we estimate the systematic error of the polarization angles to be at most $\sim 8^{\circ}$.

\section{Results}

Linear polarization was determined on several $22 \mathrm{GHz} \mathrm{H}_{2} \mathrm{O}$ maser features in both the red-

\footnotetext{
${ }^{1}$ The National Radio Astronomy Observatory (NRAO) is a facility of the National Science Foundation operated
} under cooperative agreement by Associated Universities, Inc. 


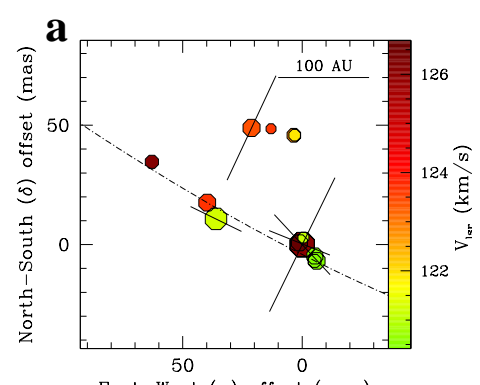

c

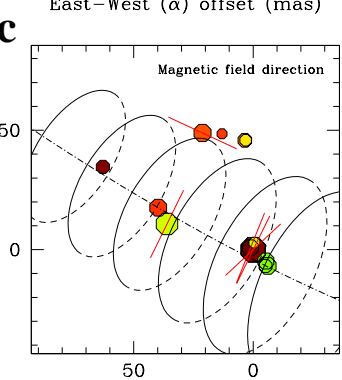

e

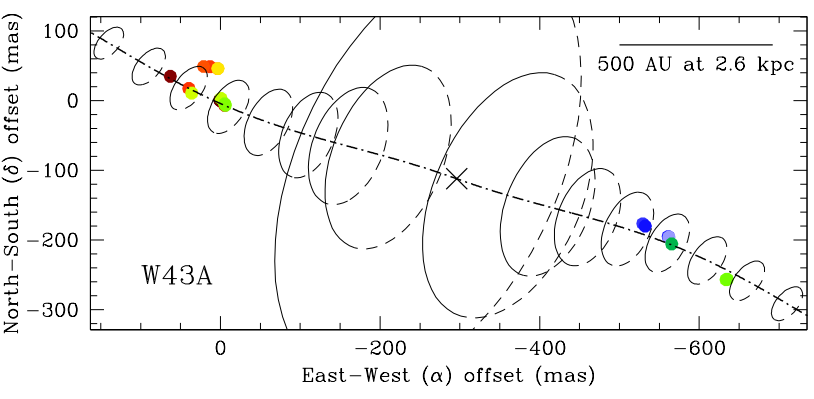

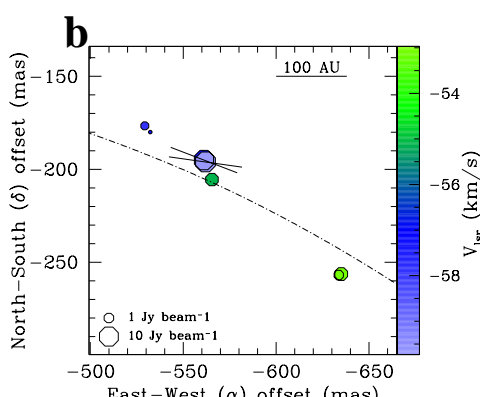

d

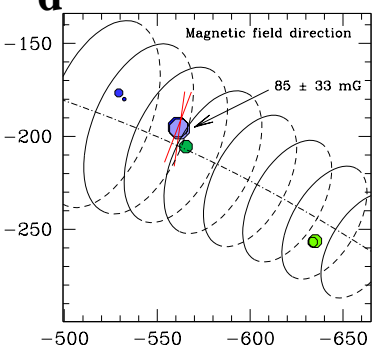

Figure 1: The $\mathrm{H}_{2} \mathrm{O}$ masers in the precessing jet (dashed-dotted line) of W43A (indicated by the cross). (a+b) The maser features with the determined linear polarization vectors scaled linearly according to the fractional linear polarization. The polarization vectors lie predominantly along the jet with a median angle of $\chi=63 \pm 12^{\circ}$ east of north. (c+d) The toroidal magnetic field of W43A. The vectors indicate the determined magnetic field direction, perpendicular to the polarization vectors, at the location of the $\mathrm{H}_{2} \mathrm{O}$ masers. The ellipses indicate the toroidal field along the jet, scaled with magnetic field strength $\propto r^{-1}$. The colour scale indicates the velocity of the maser features and the hexagonal symbols are scaled according to the maser flux density.

shifted and blue-shifted tip of the jet of W43A. The linear polarization vectors are either parallel or perpendicular to the magnetic field [19]. As discussed in V06 and elaborated upon in [20], we find that the polarization vectors of the masers of W43A are mostly perpendicular to the magnetic field direction, and that the almost exactly $90^{\circ}$ flip in polarization angle observed on the strongest maser feature is direct confirmation of current maser theory. The observed maser features and their linear polarization vectors are shown at the top of Fig.1 and the derived magnetic field direction is shown in the bottom panel of the figure. In addition to the linear polarization, we detected circular polarization of $P_{V}=0.33 \pm 0.09 \%$ (Fig. 2, left), which, as shown in V06, indicates a deprojected magnetic field strength of $B=200 \pm 78 \mathrm{mG}$.

As described in [18], the models used to determine the magnetic field from the maser total intensity and polarization spectra also yield the intrinsic maser thermal width $\Delta v_{\text {th }} \approx 0.5(T / 100)^{1 / 2}$, where $T$ is the temperature in the maser region. Additionally, the models produce the maser emerging brightness temperature $T_{b} \Delta \Omega$, where $T_{b}$ is the brightness temperature and $\Delta \Omega$ the unknown beaming solid angle. As the models give $\Delta v_{\text {th }} \approx 1.1 \mathrm{~km} \mathrm{~s}^{-1}$, we find an intrinsic temperature in the maser jet of $T \approx 500 \mathrm{~K}$. For the emerging brightness temperature we find $T_{b} \Delta \Omega \approx 8 \times 10^{9} \mathrm{~K}$ sr. Assuming a size of $\sim 0.4$ mas for the unresolved maser features, which corresponds to $\approx 1 \mathrm{AU}$, we find a brightness temperature lower limit $T_{b} \approx 1.5 \times 10^{11} \mathrm{~K}$. Thus, the upper limit on the beaming solid angle $\Delta \Omega \approx 5 \times 10^{-2} \mathrm{sr}$, which is similar to the values found in star-forming regions [18]. Fi- 

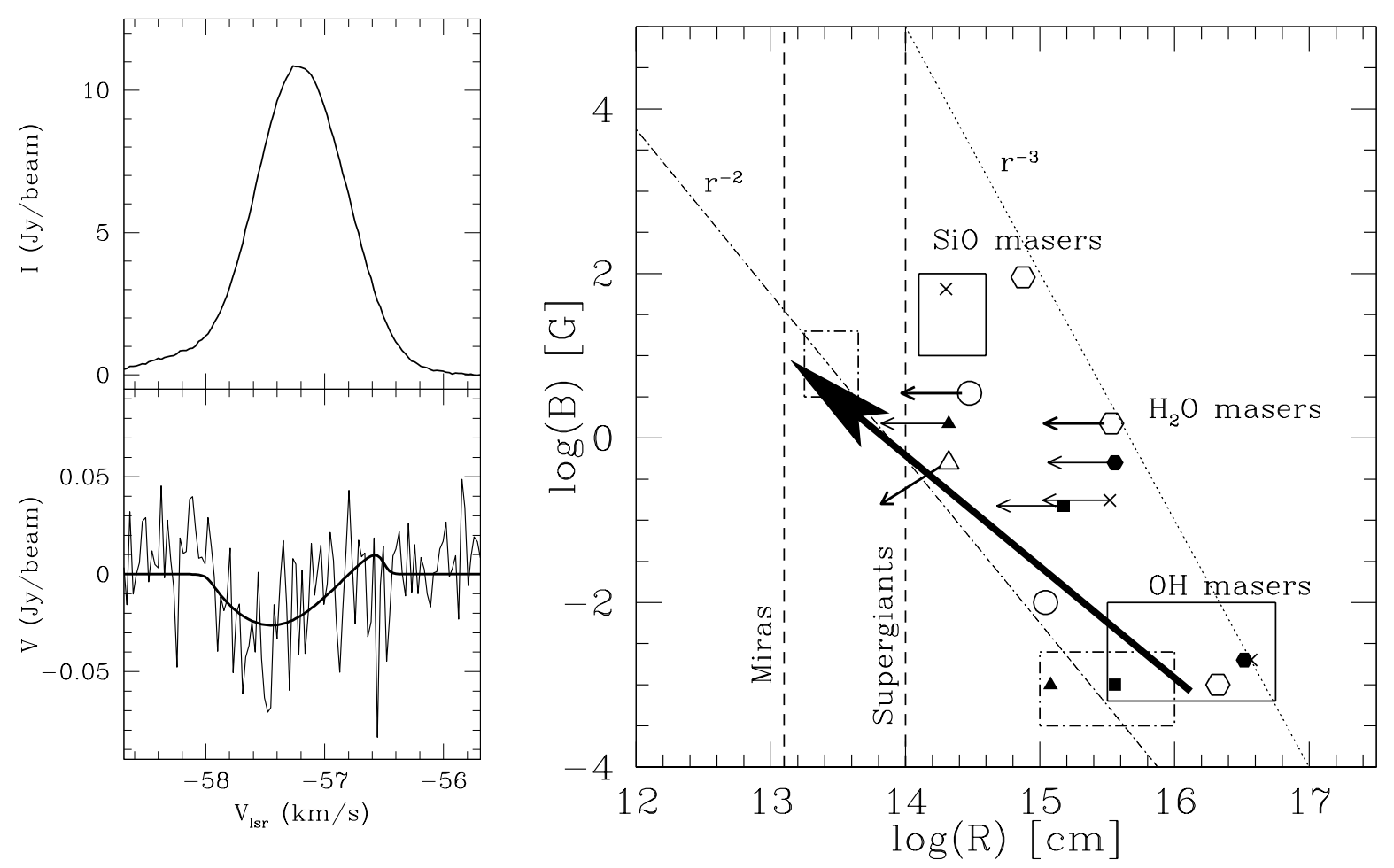

Figure 2: (left) The total intensity and circular polarization spectrum of the $\mathrm{H}_{2} \mathrm{O}$ maser feature for which circular polarization was detected. (right) The figure reproduced from [11] of measured magnetic fields on the masers in the CSEs of evolved stars. The dashed-dotted boxes indicate the range of magnetic field strengths measured on the $\mathrm{SiO}$ and $\mathrm{OH}$ masers of Mira stars and the solid boxes those of supergiant stars. The thin arrow indicate the $\mathrm{H}_{2} \mathrm{O}$ maser magnetic fields measured in [10, 11, where the length of the arrows indicate the thickness of the $\mathrm{H}_{2} \mathrm{O}$ maser shell with the symbols drawn on the outer edge. The symbols without arrows are the measurements on $\mathrm{SiO}$ and $\mathrm{OH}$ masers from the literature on the same sample of stars. The thick solid arrow indicates the toroidal magnetic field in the jet of W43A.

nally, comparing the emerging brightness temperature with the maser brightness temperature $T_{S}$ at the onset of saturation we can conclude that for $\Delta \Omega \approx 5 \times 10^{-2}$ sr the masers of W43A are mostly saturated.

\section{Discussion}

As we conclude that the $\mathrm{H}_{2} \mathrm{O}$ masers are excited in swept up material in the jet [20], the magnetic field in the maser region is enhanced. Partial coupling of the magnetic field to the gas indicates that the toroidal magnetic field strength around the collimated jet, at $\sim 1000 \mathrm{AU}$ from $\mathrm{W} 43 \mathrm{~A}$ is $B_{\phi} \sim 2 \mathrm{mG}$ (V06). The linear polarization vectors, perpendicular to the jet axis, indicate that we are tracing a confining toroidal magnetic field. A toroidal field depends on distance from the star $r$ as $B_{\phi} \propto r^{-1}$ and we thus find that at the surface of the star, the magnetic field strength is between $\sim 2-20 \mathrm{G}$. As shown in Fig. Z (right), this is fully consistent with earlier measurements of the magnetic field in the envelopes of evolved stars. $\mathrm{SiO}$ maser observations [2] seem to indicate 
that the jet formation occurs within the $\mathrm{SiO}$ maser region, close to the star, and thus that the collimating agent is related to the star itself. Considering the shape and strength of the magnetic field in the jet of W43A, we conclude that the magnetic field is the primary collimating agent, making this the first direct detection of magnetic collimation in any astrophysical jet.

\section{References}

[1] Diamond, P. J., Norris, R. P., Rowland, P. R., Booth, R. S., \& Nyman, L.-A. 1985, MNRAS, 212, 1

[2] Imai, H., Nakashima, J.-i., Diamond, P. J., Miyazaki, A., \& Deguchi, S. 2005, Ap. Lett., 622, L125

[3] Imai, H., Obara, K., Diamond, P. J., Omodaka, T., \& Sasao, T. 2002, Nature, 417, 829

[4] Imai, H., Morris, M., Sahai, R., Hachisuka, K., \& Azzollini F., J. R. 2004, A\&A, 420, 265

[5] Morris, M. R., Sahai, R., \& Claussen, M. 2003, in Rev. Mexicana AyA Conference Series, 20-22

[6] Boboltz, D. A. \& Marvel, K. B. 2005, Ap. Lett., 627, L45

[7] Kemball, A. J. \& Diamond, P. J. 1997, Ap. Lett., 481, L111

[8] Herpin, F., Baudry, A., Thum, C., Morris, D., \& Wiesemeyer, H. 2006, A\&A, 450, 667

[9] Etoka, S. \& Diamond, P. 2004, MNRAS, 348, 34

[10] Vlemmings, W. H. T., Diamond, P. J., \& van Langevelde, H. J. 2002, A\&A, 394, 589

[11] Vlemmings, W. H. T., van Langevelde, H. J., \& Diamond, P. J. 2005, A\&A, 434, 1029

[12] Blackman, E. G., Frank, A., Markiel, J. A., Thomas, J. H., \& Van Horn, H. M. 2001, Nature, 409, 485

[13] Blackman, E. G. 2004, in ASP Conf. Ser. 313: Asymmetrical Planetary Nebulae III: Winds, Structure and the Thunderbird, 401

[14] Frank, A. \& Blackman, E. G. 2004, ApJ, 614, 737

[15] Nordhaus, J., \& Blackman, E.G. 2006, MNRAS, 370, 2004

[16] García-Segura, G., López, J. A., \& Franco, J. 2005, ApJ, 618, 919

[17] Vlemmings, W. H. T., Diamond, P. J., \& Imai, H. 2006, Nature, 440, 58 (V06)

[18] Vlemmings, W. H. T., Diamond, P. J., van Langevelde, H. J., \& Torrelles, J. M. 2006, A\&A, 448, 597

[19] Goldreich, P., Keeley, D. A., \& Kwan, J. Y. 1973, ApJ, 179, 111

[20] Vlemmings, W. H. T., Diamond, P. J. 2006, ApJ, 648, L59 